SIOVILSJOUJSILLL

Of

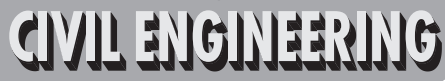

Vol. XX, 2012, No. 1, 10 - 20, DOI: 10.2478/v10189-012-0002-y

\section{METHODOLOGY FOR CONFORMANCE TESTING OF SPATIAL DATA INFRASTRUCTURE COMPONENTS INCLUDING AN EXAMPLE OF ITS IMPLEMENTATION IN SLOVAKIA}

\section{ABSTRACT}

Before any spatial data infrastructure (SDI) is implemented as fully operational, many relevant testing procedures should take place. Such procedures should evaluate the compliancy level of particular SDI components against the relevant standards and implementing rules. Hence, they should ensure a high interoperability level. Many testing activities have already been performed within the implementation of the European SDI (INSPIRE - Infrastructure for Spatial Information in the European Community). Nevertheless, a common and versatile testing methodology, which is possible to use at any level of SDI realization is still lacking. This paper proposes a conformance testing methodology for selected SDI components applicable via network services for the discovery, view and downloading of data. An example of such an implementation has taken place within an environmental SDI developed by the Slovak Environmental Agency. A testing report template summarizing the results of the tests is proposed to be considered as a common template on a national level to be used within the implementation of a National Infrastructure for Spatial Information in the Slovak Republic.

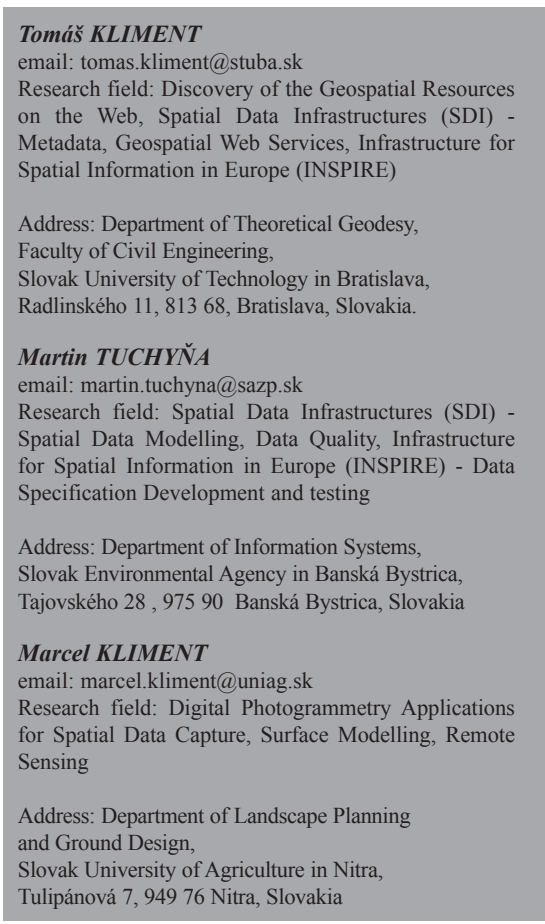

\section{KEY WORDS}

- $S D I$,

- INSPIRE,

- conformance testing,

- methodology,

- design,

- tools,

- reporting 


\section{INTRODUCTION}

Before any component of a spatial data infrastructure (SDI) can be provided for an operational phase via a Geoportal acting as a gateway to SDI (Giff, et al., 2008), several testing procedures should be performed against predefined rules to ensure the required interoperability level. Interoperability in this context is technically ensured by international standards (ISO-TC211), implementation specifications (OGC) and binding legislative documents on the European (INSPIRE 2007, 2008, 2009a, 2009b, 2010a, 2010b and 2010c) and national (Zákon 2010) levels. The fundamental components of SDI are naturally spatial data (SD), including their description, known as metadata. These components should be provided to the Geoportal (for instance, the INSPIRE Geoportal on the European level or EnviroGeoPortál on the Slovak level, for the environmental domain) by network services (NS).

The implementation phase of the INSPIRE directive began in 2010, when the first technical components were defined in order to be reported (INSPIRE Roadmap). This milestone launched activities within the European Union (EU) and member state (MS) stakeholder communities. Collaboration between the Slovak Environmental Agency (SEA) and the Slovak University of Technology (SUT) has been established to reflect the implementation process. This collaboration has been extended with the Slovak University of Agriculture (SUA) in 2011 and currently also represents Slovakia in the PTB (Persistent SDI Test Bed for Research and Education) European research community initiative. The first results gained from this collaboration were presented at the Agile 2011 international forum within a program workshop devoted to testing SDI components (Kliment, et al., 2011b).

The fundamental objective of this paper is to propose a testing methodology for SDI applicable via network services and to show an example of its implementation within the national SDI in Slovakia. Methodology section 2 introduces a technical overview of SDI components and the relations among them. Then it describes the proposed testing methodology and a design of the testing tool. A description of the tools developed and a sample testing of the SEA components is provided. The results in section 3 present a summary of the findings and propose a template for reporting them. Section 4 concludes the paper and points out future research work.

\section{MATERIALS AND METHODS}

The issue of testing SDI components has been recently defined as very important due to the implementation activities launched by the INSPIRE directive milestones. Many testing activities have been realized so far, and various works (Horak, et al., 2009; Ardielli, et al., 2009, 2011; Cibulka, 2011) are related to testing the view or also discovery (Kliment, et al., 2011a) services according to the INSPIRE requirements. The extent of the testing did not have a complex character and mainly provided information about the quality of the service, where the service's interface and content had not been tested, or if so, only at a minimal level. Other work is devoted to current data models transformation into the INSPIRE application models (Östman, et al., 2010). The ESDIN project proposed a testing environment that enables testing either NS or SD models (Portele, et al., 2011). The project Nature-SDI plus provides an INSPIRE validation briefcase for nature conservation data, which introduces methodology focused on data and metadata validation (Ford, et al., 2011). However, none of the above cited works are focused on the testing methodology for an entire SDI framework. Therefore, this paper will concentrate on such a proposed methodology in the following sections.

\subsection{OVERVIEW OF SDI COMPONENTS FROM A TECHNICAL PERSPECTIVE}

Various resources such as books, papers or cookbooks provide detailed information about SDI nowadays (Aalders, 2001; Rajabifard, 2001; Masser, 2005; Nebert 2009). It is important to mention in the beginning that many terms besides SDI now exist that are used within the geospatial information (GI) domain. Some authors use the term "Geospatial Data Infrastructure" (Brox, et al., 2002; Woldai, 2002; Groot, et al., 2000). Others use "Geospatial Information Infrastructure" (Diaz, 2010). In Slovakia, the term "Infrastructure for Spatial Information" has been established following national legislation devoted to GI, which is mainly represented by an act on the National Infrastructure for Spatial Information (Zákon,

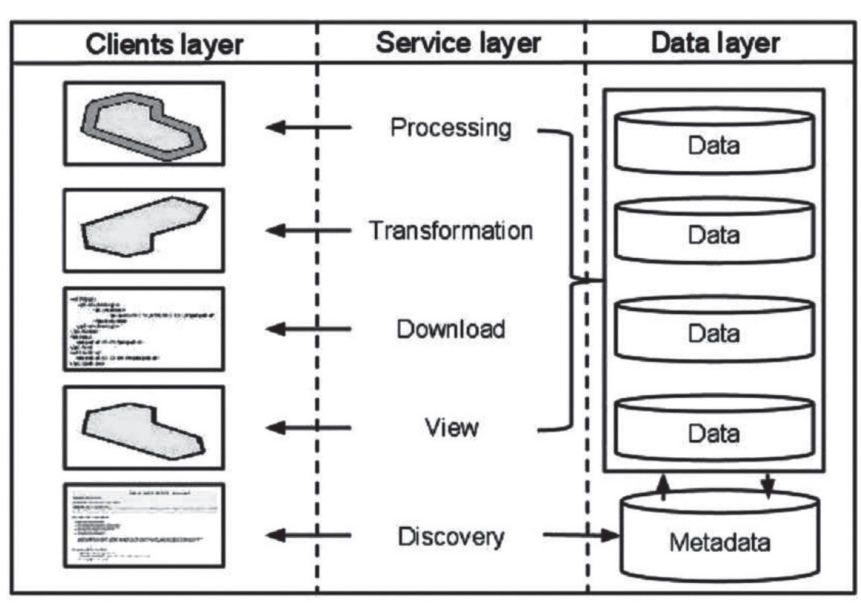

Fig. 1 SDI components from a technical perspective. 
2010). The description of an SDI is slightly different within the above- mentioned works, but they all represent the same conceptual framework. Hence we will use this term for the rest of the paper. In principle, SDI defines a framework to facilitate and coordinate data exchange and sharing among the stakeholders from various domains. SDI is much more than just the data and goes far beyond surveying and mapping. SDI provides an environment within which organisations and/or nations interact with technologies to foster activities for producing, managing and using geospatial data (Rajabifard, 2001). From a technical point of view SDI is an infrastructure that serves spatial data and its metadata (data layer) to clients (client layer) via network services (service layer) (Figure 1). The service layer of SDI defines the following types of network services:

- Discovery service - serves for data discovery based on metadata. The user specifies the search criteria (keywords, spatial/temporal extent, topic category, etc.); the service searches records in the catalogue (Kliment, 2010c) and returns records matching the criteria. The user evaluates the data concerning its quality, lineage, resolution, use constraints, access restrictions, etc., based on the metadata returned. Finally, a dataset is chosen for further access and use.

- View service - serves to portray the data as a raster set of images (maps) and provides such basic functions as zoom, pan, and legend portrayal.

- Download service - serves for downloading data to local storage in the selected data exchange format.

- Transformation service - serves for data transformation between coordinate systems or among different data models.

- Processing services - serves for various geo-processes to be invoked, such as, for instance, spatial analysis (buffer, intersect, union, etc.), or chaining several network services.

Above described technical components are standardized with a series of standards and implementation specifications both within the GI domain (ISO-TC211, OGC) and the Web domain (W3C). Further non-technical SDI components are agreements on the data sharing that play a very important role and are defined within INSPIRE (INSPIRE 2010c). Continuous monitoring of measurements is a component that implies reporting whether particular components are fulfilling requirements or not (INSPIRE 2009b). The proper deployment of SDI components, together with well-established procedures for further maintenance and development, can ensure the achievement of a high level of interoperability and attract all the other stakeholders to enlarge the SDI beyond the public sector domain (Proposal for INSPIRE Maintenance and Implementation 2011). To ensure this, relevant tests should be done. It is recommended to perform relevant tests before the operational phase of the infrastructure is launched.

\subsection{TESTING METHODOLOGY FOR SDI COMPONENTS}

The proposed methodology is based on a user scenario, where the testing tool is a remote SDI client accessing data and metadata via network services. This means that conformity of the network services is tested first and then the content itself (data and metadata). The following points of the testing methodology were proposed for the purposes of testing SDI components against legislation (INSPIRE) and related standardization (ISO, OGC):

- Testing requirements - analysis of the requirements defined in the related documentation - e.g., within the INSPIRE framework, this comprises a study of the INSPIRE directive, regulations for individual components of the infrastructure, and technical guidelines. Obviously, related ISO standards and the OGC implementation specifications have to be analysed since they define the base for INSPIRE.

- Testing scope - whether to perform a complex testing model to test all the requirements defined in the previous point the service interface (operations and the parameters of the request and response validations), quality of the service (QoS) (evaluating the defined quality parameters), service content (data and metadata validation) and other criteria, or to perform a partial testing model based on the definitions driven by the tester.

- Temporal extent - defines the time span and repetition frequency of tests which might be either long-term testing in order to evaluate the QoS precisely or short term for a fast initial overview of the service interface and the validity of its content.

- Testing scenarios - conceptual design (text description or business process models (with Business Process Modelling Notation (BPMN)), or activity diagrams (with Unified Modelling Language (UML) notation) and implementation of individual testing scenarios, depending on the testing environment. It would be ideal to have a common tool that would enable execution of all the tests.

- Testing execution and reporting results - includes the execution of individual scenarios defined in the previous point and generation of testing reports. It is important to define the structure of the report, because it is the main objective of the testing. For instance, such information as description of the testing scenarios, information about the tester, the testing environment and the main testing results (e.g. Passed/Failed, Passed at 54\%, etc.) should be included.

\subsection{DESIGN OF THE TESTING ENVIRONMENT}

The proposed testing environment is designed using UML use case diagram notation (Kanisova, et al., 2004). The individual types of actors and the corresponding use cases are shown in Figure 2. 


\section{IIOYASS JOUSSIAL \\ of $f^{*}$

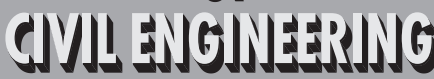

2012/1 PAGES $10-20$

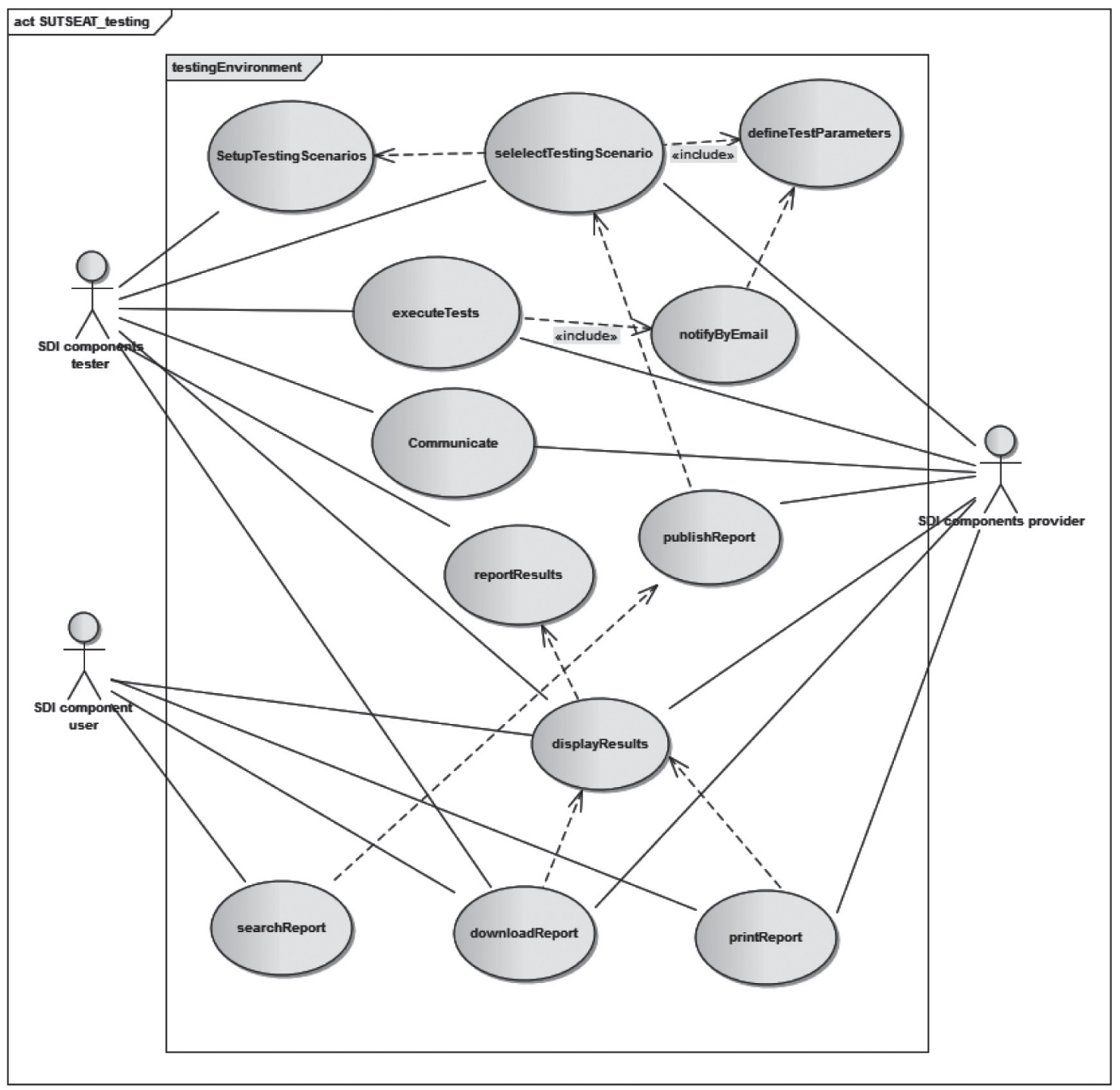

Fig. $2 U M L$ use case diagram of the testing environment.

The use case diagram defines three roles, i.e., the SDI components tester, the SDI components provider, and the SDI component user. The SDI component user role might be anyone who needs to use SDI components (i.e., a ministry, agency or citizen). Under certain circumstances the SDI component tester role and SDI component provider role may be assigned to one person. The model defines the following use cases:

- setupTestingScenario - the SDI component tester develops particular testing scenarios in order to define parameters for the individual requirements to be tested. An example is the search criteria test for the INSPIRE discovery service, where the testing environment has to send a series of DiscoverMetadata (INSPIRE 2009a) requests with a particular search criterion against the metadata in the catalogue and validate the server response.
- selectTesting Scenario - this use case includes defineTestParameters use case for the SDI component provider and tester. It defines main parameters necessary for the testing, as the service URL definition, temporal coverage of testing, email notification and others.

- executeTest - the SDI component tester or SDI component provider role launches the test defined in the previous use cases. The use case notifyByEmail may provide both notifications about the beginning and completion of the tests.

- reportResults - the SDI component tester browses all the results, including the technical details (the content of responses with errors, etc.) and defines the final structure of the report concerning its receiver (SDI component provider or user). 
- publishReport - the SDI component provider publishes a report in order to promote the results to a third party (SDI component user).

- displayResults - all the roles can display the report within the GUI application and in the structure(s) defined within the reportResults use case.

- downloadReport - all the roles can download the report in a predefined format (e.g. HTML, XML, or PDF).

- printReport - the SDI component provider and user print out the testing report.

- communicate - the SDI component tester and SDI component provider communicate mutually using the communication interface for consultations related to configuration, execution, results and reporting of the individual tests.

- searchReport - the SDI component user queries the testing tool database in order to display the particular testing report of the SDI components he wants to use.

\subsection{TESTING TOOLS}

For testing purposes based on the above-presented methodology, testing tools currently available on the scene as well as tools developed in-house at the Department of Theoretical Geodesy (DTG) of SUT were used. External tools used for testing the discovery and view service are described in detail within the publications (Kliment, 2010b; Kliment, et al., 2011a).

This section aims to provide an overview of the tools developed at DTG. The first one webtest v. 1.0 (Fig.3) is written in the Java programming language and also uses Java Server Pages (JSP) technology for a fast way to create dynamic web content. The webtest provides the functionality of sending single, multiple and simultaneous Hypertext Transfer Protocol (HTTP) GET and POST requests and takes measurements for two types of response times:

- the time between sending the request and the first downloaded byte of the response - INSPIRE response time (INSPIRE 2009a)

- the time between sending the request and the last downloaded byte of the response

The tool provides a simple graphic user interface (GUI), where the individual testing scenarios may be imported either with a copy and paste of the XML fragment defining the request, or directly uploading the entire XML configuration file containing the complex testing scenario. The webtest is a web tool; thus, any users may use it remotely by using a web browser without installation of any additional software to their computers. Another useful function is to check whether the predefined string appears in the response or not; if so, its appearance is reported. This function is used to identify the expected results using, for instance, a character string within the

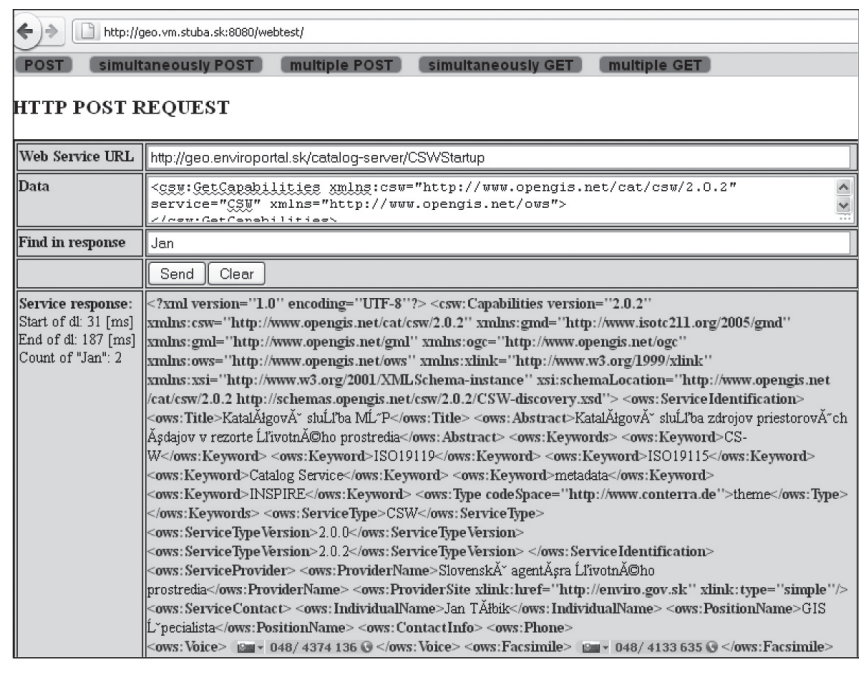

Fig. 3 GUI testing tool webtest - simple HTTP POST request/ response.

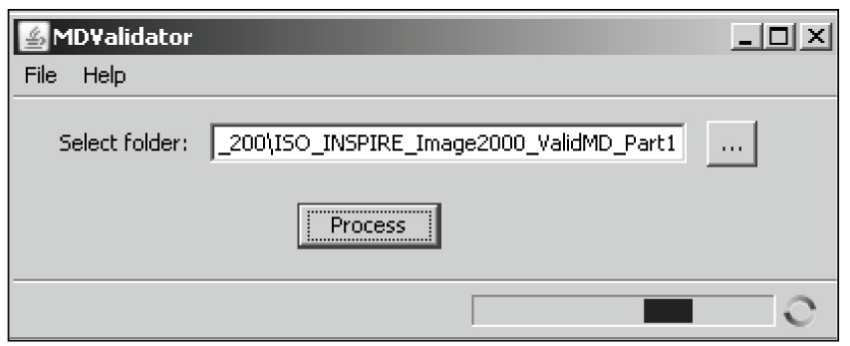

Fig. 4 GUI validation tool MDValidator - batch validation execution.

unified resource identifier (URI). The testing results are shown in the table with the following information: request identifier, response times as described above, and the number of strings discovered in the response. An example of the table can be seen in the results section. The tool provides neither any graphic statistics in the form of plots insofar as they would be used for long term testing, nor storage for the testing results. Nevertheless, this is part of the on-going development of the webtest v. 2.0, which is planned to be finalized during the first half of the year 2012 (Cibulka, 2012). The second tool is a MDValidator (Fig. 4), which performs batch metadata validation against the INSPIRE requirements (INSPIRE, 2008). The main reason for developing such a tool was its absence from the metadata tools available on the scene.

The MDValidator incrementally reads metadata in the XML format from the local directory and sends them to an online validation service (INSPIRE validation service). The result is a report in the 


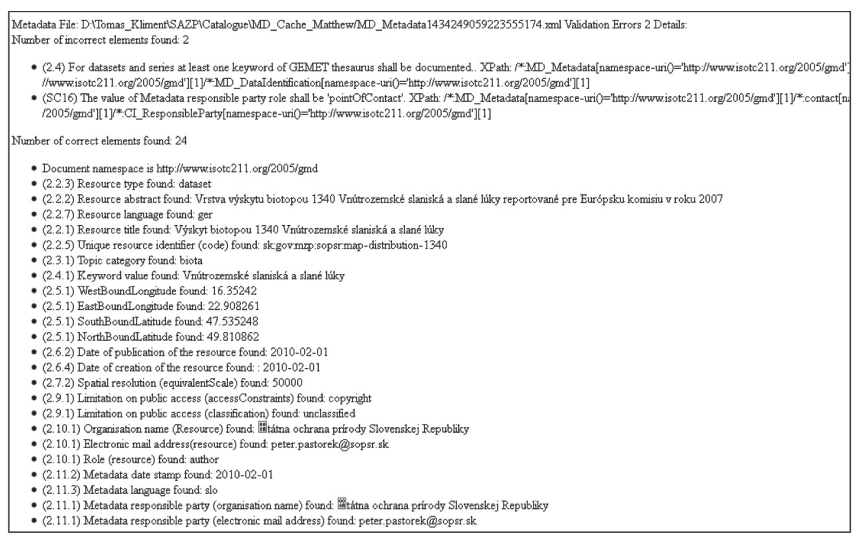

Fig. 5 Fragment of validation report from the MDValidator.

HTML/XML format (Fig. 5), which summarizes the validation results - the name of the validated metadata record and its path, number and listing of correct/incorrect elements found. The validation service does not perform a validation against the ISO metadata schema (ISO 2003, 2007); what is actually its drawback. This is an important aspect to consider, because if a metadata record is valid against INSPIRE, it does not have to be valid against the ISO schema and vice versa.

\subsection{OVERVIEW OF THE SDI COMPONENTS PROVIDED BY SEA}

The SEA provides spatial data and metadata via network services listed in Tab. 1 for INSPIRE and the national infrastructure. These spatial data refer to themes defined in the annexes of the INSPIRE directive (INSPIRE, 2007) and has been provided by SEA for testing purposes:

- Annex I - metadata and spatial data for the INSPIRE spatial data themes 8 . Hydrography and 9 . Protected sites.
- Annex II - metadata and spatial data for INSPIRE spatial data theme 2. Land Cover.

- Annex III - spatial data for the INSPIRE themes 17. Biogeographical regions, 18. Habitats and Biotopes and 19. Species Distribution.

These services will be provided to the national infrastructure and connected via the operational National Geoportal in Slovakia. The user will be able to search metadata and evaluate and use spatial data via this central point of the national infrastructure under predefined conditions.

\subsection{TESTING METHODOLOGY - SAMPLE IMPLEMENTATION}

The initial testing of the discovery service provided by SEA was performed in the year 2010, and the results were presented (Kliment 2010a; Kliment 2010b). This subsection describes the realization of the testing on the discovery, view and download services introduced in the previous subsection. The main aim is to demonstrate a sample implementation of the proposed methodology under real world conditions.

\section{Testing the discovery service}

- Testing requirements - INSPIRE directive framework - the implementing rules for network (discovery) services (INSPIRE 2009a) defines four operations for the discovery service with related parameters, 27 search criteria and three QoS parameters; the implementing rules for metadata (INSPIRE 2008) defines 19 metadata elements (16 mandatory and 3 conditional) for dataset and series resources and 17 elements (13 mandatory and 4 conditional) for services. The technical guidance for the implementation of INSPIRE discovery services (INSPIRE 2011a) might assist in a better understanding of the requirements from a technical perspective.

- Testing scope - Three operations have been tested (GetDiscoveryServiceMetadata, DiscoverMetadata and

Tab. 1 List of network services provided by SEA to INSPIRE and national infrastructure.

\begin{tabular}{|c|c|c|c|}
\hline NETWORK SERVICE TYPE & INSPIRE ANNEX I & INSPIRE ANNEX II & INSPIRE ANNEX III \\
\hline \multirow{2}{*}{ DISCOVERY SERVICE } & YES & YES & NOT YET \\
& (TERRA CATALOG CSW & (TERRA CATALOG CSW & Y.0.2) \\
\hline \multirow{2}{*}{ VIEW SERVICE } & $2.0 .2)$ & YES & YES \\
& (ARCGIS SERVER WMS 1.3) & (ARCGIS SERVER WMS 1.3) & (ARCGIS SERVER WMS 1.3) \\
\hline \multirow{2}{*}{ DOWNLOAD SERVICE } & YES & YES & YES \\
& (ARCGIS SERVER WFS 1.1) & (ARCGIS SERVER WFS 1.1) & (ARCGIS SERVER WFS 1.1) \\
\hline
\end{tabular}


PublishMetadata); 23 search criteria and 2 quality parameters (performance and capacity) have been tested. The metadata has been validated against the INSPIRE (INSPIRE validation service) and ISO schema (ISO 2007).

- Temporal extent - short-term testing; every testing scenario was executed repeatedly within a short period (3 days)

- Testing scenarios - (1) scenario configured for the testing of the search criteria together with the DiscoverMetadata operation and performance of the QoS parameter. (2) Scenario for testing the combinations of operations defined in the scope and capacity of the QoS parameter. (3) Scenario for the testing of PublishMetadata operation (Push and Pull mechanism as defined in INSPIRE, 2009).

- Testing tools - webtest and MDValidator.

\section{Testing the view service}

- Testing requirements - INSPIRE directive framework the implementing rules for the network services (INSPIRE 2009b) define three operations for the view service with related parameters and three QoS parameters. The technical guidance for the implementation of the INSPIRE view services (INSPIRE 2011b) might assist in a better understanding the requirements from a technical perspective.

- Testing scope - two operations have been tested (GetViewServiceMetadata and GetMap) and two quality parameters (performance and capacity). The request and response parameters have not been tested.

- Temporal extent - short-term testing; every testing scenario was executed repeatedly within a short period (3 days)

- Testing scenarios - (1) scenario configured for testing the GetMap operation in a combination of all the layers provided by the SEA view services and the QoS parameter performance. (2) Scenario for testing the combinations of the operations defined in the scope and capacity of the QoS parameters.

- Testing tools - webtest.

\section{Testing the download service}

- Testing requirements - INSPIRE directive framework - the implementing rules for network services (INSPIRE 2010b) defines four operations for pre-defined dataset download service and another two for direct access download operations plus their parameters and three QoS parameters. The service content (data) applies implementing rules for the spatial data sets (INSPIRE 2010a) and the technical guidance and Geography Markup Language (GML) schemas for the individual INSPIRE spatial themes.

- Testing scope - three operations have been tested (GetDownloadServiceMetadata, GetSpatialDataSet and
DescribeSpatialDataSet) and two network service quality parameters (performance and capacity). The request and response parameters have not been tested. The data has not been validated against the INSPIRE schemas developed at this phase.

- Temporal extent - short-term testing; every testing scenario was executed repeatedly within a short period (3 days)

- Testing scenarios - (1) scenario configured for testing the GetSpatialDataSet in combinations of the data provided by the SEA download services (Table 1) and the performance and capacity of the QoS parameters. (2) Scenario for the testing combinations of operations defined in the scope above and the QoS parameter performance.

- Testing tools - webtest.

\section{RESULTS}

The testing results provided by the webtest and the MDValidator are indeed not very user friendly. An example of the results table created by the webtest is shown in Figure. 6. This table displays the results from the testing scenario (1) of testing the discovery service. The results table above provides IDs of the individual requests (second column from the left) sent within the scenario, the response times in milliseconds (third and fourth columns from the left), and the number of predefined strings discovered in the responses (the right column). This table provides raw results

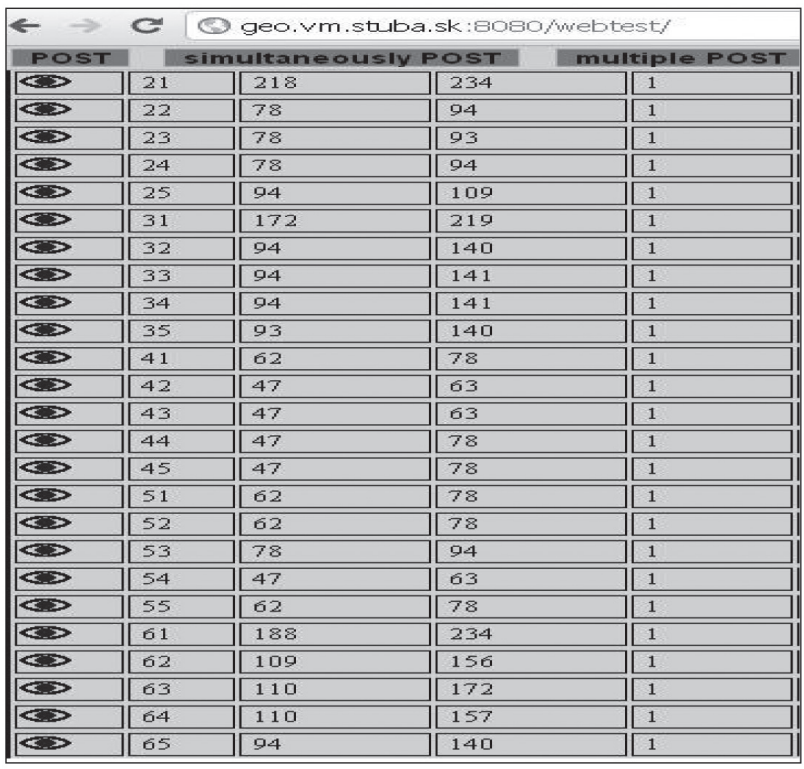

Fig. 6 webtest testing report fragment - discovery service, scenario (1). 
2012/1 PAGES $10-20$

Tab. 2 Testing results - proposed template structure for the report.

\begin{tabular}{|c|c|c|c|}
\hline TESTED SERVICE & DISCOVERY SERVICE & VIEW SERVICE & DOWNLOAD SERVICE \\
\hline $\begin{array}{l}\text { INTERFACE } \\
\text { (OPERATIONS\&PARAMETERS) }\end{array}$ & \multicolumn{3}{|c|}{ RESULTS } \\
\hline GetNetworkServiceMetadata & $\begin{array}{c}\text { Supported } \\
\text { Parameters not tested }\end{array}$ & $\begin{array}{c}\text { Supported } \\
\text { Parameters not tested }\end{array}$ & $\begin{array}{c}\text { Supported } \\
\text { Parameters not tested }\end{array}$ \\
\hline DiscoverMetadata & $\begin{array}{c}\text { Supported } \\
\text { Parameters not tested }\end{array}$ & & \\
\hline PublishMetadata & $\begin{array}{c}\text { Supported } \\
\text { Parameters not tested }\end{array}$ & & \\
\hline LinkService & Not tested & Not tested & Not tested \\
\hline GetMap & & $\begin{array}{c}\text { Supported } \\
\text { Parameters not tested }\end{array}$ & \\
\hline GetSpatialDataSet & & & $\begin{array}{c}\text { Supported } \\
\text { Parameters not tested }\end{array}$ \\
\hline DescribeSpatiaDataSet & & & $\begin{array}{c}\text { Supported } \\
\text { Parameters not tested }\end{array}$ \\
\hline GetSpatialObject & & & Not tested \\
\hline DescribeSpatialObject & & 2 & Not tested \\
\hline QUALITY OF SERVICE & \multicolumn{3}{|c|}{ RESULTS } \\
\hline Performance & $\begin{array}{l}\text { Satisfied }(115 \text { requests sent } \\
115 \text { responses }<3 \mathrm{~s})\end{array}$ & $\begin{array}{c}90 \% \text { Satisfied }(30 \text { requests } \\
\text { sent } 27 \text { responses }<5 \text { s })\end{array}$ & $\begin{array}{l}\text { Satisfied }(30 / 10 \text { requests sent } \\
30 / 10 \text { responses }<10 / 30 \text { s) }\end{array}$ \\
\hline Capacity & $\begin{array}{c}\text { Satisfied } \\
(30 \text { simultaneous requests } \\
\text { sent } 30 \text { responses }<3 \text { s })\end{array}$ & $\begin{array}{c}70 \% \text { Satisfied } \\
(20 \text { simultaneous requests sent } \\
14 \text { responses }<5 \mathrm{~s})\end{array}$ & $\begin{array}{c}\text { Satisfied } \\
(10 \text { simultaneous requests sent } \\
10 \text { responses }<30 \mathrm{~s})\end{array}$ \\
\hline Availability & Not tested & Not tested & Not tested \\
\hline OTHER CRITERIA & \multicolumn{3}{|c|}{ RESULTS } \\
\hline Search criteria & Supported (tested 23/27) & & \\
\hline $\begin{array}{l}\text { Search Criteria for the Get Spatial } \\
\text { Object Operation }\end{array}$ & & & Not tested \\
\hline SERVICE CONTENT & \multicolumn{3}{|c|}{ RESULTS } \\
\hline Metadata models & $\begin{array}{l}\text { Valid (validated } 161 \mathrm{MD} \\
\text { records against INSPIRE and } \\
\text { ISO) }\end{array}$ & & \\
\hline Data models & 2 & 2 & Not tested \\
\hline
\end{tabular}

that probably would not say very much to the SDI component user. Therefore, a report template, which will aggregate and summarize all the information into a more humanly readable and understandable way, should be proposed and implemented. As a result of this work, the following structure has been proposed (Table 2) and filled in with the results gained within this implementation example. The main aim is to create a template to be used within the national infrastructure.
The proposed template consists of a table header defining the tested network services and four main blocks according to the testing scope defined within the methodology. The first block provides the interface's testing definition and the results for the services tested. The second block reports QoS, and the third reports any other criteria defined within the requirements. The last, but not least, block reports on the validity of the service content. Values such as Supported, Supported/Parameters Tested and Not Tested 
are used to evaluate the service interface part. Values Satisfied, X\% Satisfied and Not Tested are used for the QoS assessment. Finally, the Supported and Not Tested values are used for other criteria and Valid, Invalid, Not Tested for the service content validation.

\section{CONCLUSIONS AND FUTURE WORK}

The work presented within this paper provides proposals for a methodology to verify the conformity of existing SDI components with the requirements defined for the SDIs established on the European level. An example of the implementation of the proposed methodology has been provided as well. Nevertheless, this example was not meant to constitute comprehensive testing against the SEA network service, but rather to check the feasibility of the proposed methodology. The report structure has been proposed accordingly. Moreover, collaboration between the public and academic sector organisations has been proved as very important to reach a common goal - interoperable access to spatial information among the stakeholders from the entire society. The methodology and an example of its implementation showed the demands for appropriate knowledge and understanding of all the requirements defined by the framework establishing SDI. Preparation of the testing scenarios is a very important part of the methodology and is directly related to the testing coverage. It has been discovered that it consumes a significant amount of time to prepare them in a proper way. The testing results should be easily interpreted and aggregated in an understandable way. Moreover, if possible, various appropriate levels of compliancy should be introduced. The results presented confirm the merits of an initiative such as the Persistent Test Bed (PTB) and, at the same time, introduce motivation for future research work. We would like to address future work by the following:

- Discussions, proposals, and suggestions on the testing methodology presented within the expert groups dealing with testing on both the European and national levels.

- Testing scenario extensions by the following: (1) For all the requirements defined by INSPIRE, or by specific national infrastructure ones in the future. (2)The parameters of the operations have to be validated against INSPIRE's specific constraints (e.g. The INSPIRE network service shall provide INSPIRE compliant metadata within the GetNetwotkServiceMetadata response, language parameter, layer metadata for the view service, spatial dataset metadata for download service (INSPIRE 2009a, 2010b), etc. (3) Preparation and execution of long-term tests for estimating accurate service quality parameters. (4) Documentation of individual scenarios on a conceptual level (UML activity or BPMN diagrams, text descriptions).

- Testing report template - discussions and decisions on the final form and content of the reports with a focus on their target purpose (e.g., a tabular form with information such as date, test description, test execution, results, pass/fail definitions, comments, comparability of reports).

- The extension of the testing tool by the following: (1) Functions for reporting exports, statistical calculations, plots. (2) Implementation of the testing scenario series as complex tests (e.g. INSPIRE discovery service testing scenario, etc.) (3) Results stored in the form of a database to avoid the loss of results in long-term testing and support querying functionality.

- The testing of the service content: (1) Validating the metadata against the ISO metadata model (ISO 19115, 19139) and the specific constraints of the INSPIRE metadata regulation. (2) Validating spatial data against GML application schemas defined within particular INSPIRE data specifications.

New legislative requirements as well as user requirements in the field of GI additionally bring about new responsibilities as well as new challenges and space for the exchange of know-how in order to achieve their fulfilment. An important part includes efforts to promote testing (increasing awareness). Moreover, a proposal for the establishment of a common testing platform allowing sharing tools, materials, methodologies, experience and expertise related to testing SDI components is very important. Searching for solutions to particular tasks and open issues connected with building information infrastructures provides new opportunities for collaboration among bodies. This allows the opening up of new possibilities for the establishment of more intensive communication among the bodies that have previously not been cooperating. The authors of this paper believe that the experience presented here will also provide inspiration for the extension of such collaborations or eventually to create similar initiatives in other domains on several levels from national, regional and international ones. 


\section{REFERENCES}

[1] Aalders Henri J. G. L., Harold Moellering (2001). Spatial Data Infrastructure.

[2] Ardielli J., Horak J.,Ruzicka J. (2011). View Service Quality Testing According to INSPIRE Implementing Rules. Elektronika ir elektrotechnika. - Kaunas: Technologija, Lithuania, 2011 - 6 pp. In print.

[3] Ardielli J., Horak J. (2009). The Testing of the Web Services Accessibility of CENIA Company. Proceedings of GIS Ostrava 2009. - VŠB-TU, Ostrava, Czech Republic, 2009 - 8 pp. ISBN 978-80-87294-00-0.

[4] Brox, C., Bishr, Y., Kuhn, W., Senkler, K., \& Zens, K. (2002). Toward a Geospatial Data Infrastructure for North RhineWestphalia. Computer, Environment and Urban Systems, pp. 19-37.

[5] Cibulka, D: (2011). Testing of the view services. Proceedings of Juniorstav 2011 - VUT Brno, Czech Republic epublic, 2011- 10 pp. ISBN 978-80-214-4232-0 (in Slovak).

[6] Cibulka, D. (2012). Publication of selected geodata in a web environment. Proceedings of Juniorstav 2012 - VUT Brno, Czech Republic, 2011 - 6 pp. ISBN 978-80-214-4393-8 (in Slovak).

[7] Díaz L. (2010). Improving resource availability for Geospatial Information Infrastructures. Dissertation thesis. University of Jaume I of Castellón, Spain. 2010

[8] Ford M., Martirano G., Schleidt K. and Vinci F. (2011). An INSPIRE validation briefcase for nature conservation and beyond. INSPIRE Conference 2011, Edinburgh, Scotland. 2011.

[9] Giff G.,Van Loenen B., Crompvoets J. and Zevenbergen J. (2008). Geoportals in Selected European States: A NonTechnical Comparative Analysis. GSDI-10, St. Augustine, Trinidad, $2008-14$ pp.

[10] Groot, R. and McLaughin, J., (eds.) (2000). Geospatial Data Infrastructure: Concepts, cases and good practice. Oxford University Press. 2000.

[11] Horak J., Ardielli J., Horakova B. (2009). Testing of Web Map Services. International Journal of Spatial Data Infrastructures Research - Rotterdam, 2009. - 19 pp. ISSN 1725-0463

[12] INSPIRE (2007). Directive 2007/2/EC of the European Parliament and of the Council of 14 March 2007 establishing an Infrastructure for Spatial Information in the European Community (INSPIRE)
[13] INSPIRE (2008). Commission Regulation (EC) No 1205/2008 of 3 December 2008 implementing Directive 2007/2/EC of the European Parliament and of the Council as regards Metadata

[14] INSPIRE (2009a). Commission Regulation (EC) No 976/2009 of 19 October 2009 implementing Directive 2007/2/ EC of the European Parliament and of the Council as regards the Network Services

[15] INSPIRE (2009b). Commission Decision regarding INSPIRE monitoring and reporting.

[16] INSPIRE (2010a). Commission Regulation (EU) No 1089/2010 of 23 November 2010 implementing Directive 2007/2/EC of the European Parliament and of the Council as regards Interoperability of Spatial Data Sets and Services

[17] INSPIRE (2010b). COMMISSION REGULATION (EU) No 1088/2010 of 23 November 2010 amending Regulation (EC) No 976/2009 as regards Download Services and Transformation Services

[18] INSPIRE (2010c). COMMISSION REGULATION (EU) No 268/2010 of 29 March 2010 implementing Directive 2007/2/EC of the European Parliament and of the Council as Regards the Access to Spatial Data Sets and Services of the Member States by Community Institutions and Bodies under Harmonised Conditions

[19] INSPIRE (2011a). Technical Guidance for the Implementation of INSPIRE Discovery Services

[20] INSPIRE (2011b). Technical Guidance for the Implementation of INSPIRE View Services

[21] ISO (2003). ISO 19115:2003 - Geographic information Metadata. ISO, Switzerland, 2003

[22] ISO (2007). ISO/TS 19139:2007 - Geographic information Metadata - XML schema implementation, ISO, Switzerland, 2007

[23] Kanisová H., Muller M. (2004) - UML srozumitelně. Computer Press, Brno, 2004. ISBN 80-251-0231-9 (in Czech).

[24] Kliment T. (2010a). Discovery service testing according to INSPIRE implementing rules -. Abstract in proceedings of GI2010 - X - Border SDI/GDI - Symposium, Dresden, Germany, 2010. ISSN 1801-6480

[25] Kliment T. (2010b). Design and pilot realization of the testing process for the INSPIRE discovery service. ENVIRO-IFORUM 2010, Zvolen, Slovakia, ISBN 978 - 80 - 88850 - 96 - 0. pp 38-42 (in Slovak). 


\section{REFERENCES}

[26] Kliment T. (2010c). Metainformation infrastructure for geospatial information. Slovak Journal of Civil Engineering, Slovak University of Technology in Bratislava, Slovakia, 2010 -7 pp. ISSN 1210-3896

[27] Kliment T., Cibulka D. (2011a). Discovery and view service testing according to the INSPIRE requirements. Proceedings of GIS Ostrava 2011 - VŠB-TU Ostrava Czech Republic, 2011 - 9 pp. ISBN 978-80-248-2366-9 (in Slovak).

[28] Kliment T., Cibulka D., Tuchyna M., Kliment M., Koska M., Mozolik P., Tobik J. (2011b). Use case of public and academic sector organization cooperation related to SDI component testing. Presentation at Workshop Testbed Research PTB, AGILE 2011, Ultrecht, The Netherlands, 2011 $-23 \mathrm{pp}$.

[29] Kliment T., Tuchyna M., Kliment M. (2011c). Testing of SDI components - A fundamental interoperability element within INSPIRE and and National SDI's. Abstract in proceedings of GI2011 - X - Border SDI/GDI - Symposium, Dresden, Germany, 2011. ISSN 1801-6480.

[30] Masser Ian (2005). GIS Worlds: Creating Spatial Data Infrastructures. ESRI Press, New York, USA. 2005. ISBN 1-58948-122-4.

[31] Nebert, D. (2009). Developing Spatial Data Infrastructures: The SDI Cookbook. Version 3.0, Global Spatial Data Infrastructure, $2009-150 \mathrm{pp}$.

[32] Östman A. (2010). Network for testing GI services. Proceedings of GIS Ostrava 2010. VŠB-TU Ostrava, Czech Republic, 2010 - 6 pp. ISBN 978-80-248-2171-9.

[33] Portele C., Östman A., Koutroumpas M., He X., Kovanen J., Schneider M., Skopeliti A. (2011). Testing - an essential aspect of establishing an SDI. INSPIRE conference 2011 Edinburgh, Scotland. 2011.

[34] Rajabifard, A. and I.P.Williamson (2001). Spatial Data Infrastructures: Concept, SDI Hierarchy and Future directions. GEOMATICS' 80 Conference. Tehran, Iran.
[35] Woldai, T. (2002). Geospatial Data Infrastructure: The problem of developing metadata for geoinformation in Africa. In: Proceedings in 4th AARSE Conference on geoinformation for sustainable development in Africa. October 14-18 2002 Abuja, Nigeria.

[36] Zákon (2010). Law No. 3/2010 on the National Infrastructure for Spatial Information (in Slovak).

Internet resources:

[37] BPMN - Business Process Model and Notation - http://www. omg.org/spec/BPMN

[38] EnviroGeoPortál - http://geo.enviroportal.sk/

[39] ESDIN - European Spatial Data Infrastructure with a Best Practice Network - http://www.esdin.eu/

[40] gmd - Geographic MetaData extensible markup language - http://standards.iso.org/ittf/Publicly Available Standards/ ISO_19139_Schemas/gmd/gmd.xsd

[41] INSPIRE Geoportal - http://www.inspire-geoportal.eu/

[42] INSPIRE Roadmap - http://inspire.jrc.ec.europa.eu/index.cfm/ pageid/44

[43] INSPIRE validation service - http://www.inspire-geoportal.eu/ INSPIREValidatorService/resources/validation/ inspire

[44] ISO-TC211 - ISO/TC 211 Geographic information/Geomatics - http://www.isotc211.org/

[45] OGC - Open Geospatial Consortium - http://www. opengeospatial.org/

[46] Persistent SDI Test Bed for Research and Education - http:// sdi-testbed.eu/

[47] Proposal for INSPIRE Maintenance and Implementation - http://ec.europa.eu/transparency/regcomitology/index. $\mathrm{cfm}$ ?do=search.documentdetail\&oA3xqr5242jcjc4gaV9U5M1 PeKEWYLiu+kATHKRP84QxdbQ+AI/X9VTTMRqv00VG

[48] W3C - World Wide Web Consortium - www.w3.org

[49] webtest - tool for web services testing - http://geo.vm.stuba. sk:8080/webtest 